\title{
Enhancement of Degraded Manuscript Images using Adaptive Gaussian Thresholding
}

\author{
Nikunj Khetan ${ }^{1 *}$, Lakshya Kejriwal ${ }^{2}$ and Dr. S. Indu ${ }^{3}$ \\ ${ }^{1,2,3}$ Delhi Technological University \\ Inikunjkhetan123@gmail.com, ${ }^{2}$ lakshya5340@gmail.com, ${ }^{3}$ s.indu@dce.ac.in
}

\begin{abstract}
Manuscripts in physical form are easily damaged over time resulting in loss of important information. Hence, there is a need to preserve the knowledge these manuscripts hold by enhancing the readability of the damaged manuscript by applying various image analysis techniques and storing them in digital form to prevent further deterioration of manuscript information. There are multiple well-known methods for document enhancement but, they are not suitable for use in enhancing damaged manuscript images. We propose a novel method to address the problem of enhancement and binarization of degraded manuscript images that applies a dual filtering technique for noise removal, Gaussian based adaptive thresholding technique and post processing using morphological operations that enhances readability of manuscript images. Our method showed good performance on qualitative as well as quantitative evaluation performed on 27 digital manuscript images with uniform character formation and an overall pseudo F-measure of $60.12 \%$. Furthermore, our method is also compared with other well-known document enhancing techniques to establish the better applicability of our technique to preservation of manuscript information.
\end{abstract}

Keywords: Manuscript, Gaussian, Thresholding, Binarization, Enhancement

\section{Introduction}

Ancient manuscripts contain information pertaining to fields like art, science, astronomy, astrology, architecture, law, music and medicine. They are an important part of our cultural heritage. Parchments and palm leaf have been generally used as materials for manuscript writings but the ravages of time have affected legibility of characters in ancient manuscripts in the form of ink bleeding, smears, blotches, etc. There is a lot of ongoing eff ort to preserve manuscripts in physical form to prevent further deterioration but this has resulted in restricted access to the public. Hence, there is a need to store manuscripts in an enhanced digital form. Enhancement of manuscript images is an important digital heritage application and has many researchers working on methods to enhance and binarize historical documents. A lot of work has been done to enhance digital images of historic documents and binarize them so that OCR readability is feasible. The main objective is to separate foreground (text layer) from degraded(noisy) background layer and binarize the document so that readability is significantly enhanced.

Most commonly used method is the thresholding technique that separates these layers. Non-thresholding techniques [1][2][3] have also been used but, have been seen to show less promising results as compared to thresholding techniques [4]. Otsu [5], Kapur [6] and Kittler [7] use global thresholding technique in which a single threshold value is used to segment foreground and background.

Bernsen's method [8] computes an adaptive threshold by using the maximum and minimum gray-level value of the local block. The local contrast is given by:

$$
C(i, j)=I_{\max }(i, j)-I_{\min }(i, j)
$$


Where $C(i, j)$ denotes the contrast of an image pixel $(i, j), I_{\max }(i, j)$ and $I_{\min }(i, j)$ denote the maximum and minimum intensities within a local neighborhood windows of $(i, j)$, respectively. If the local contrast $C(i, j)$ is smaller than a threshold, the pixel is set as background. Otherwise it is classified as text or background after comparing with the mean of $I_{\max }(i, j)$ and $I_{\min }(i, j)$. This method fails to work properly on degraded documents with complex background.

Local thresholding techniques used by Niblack [9] and Sauvola [10] calculate threshold value for a local block. Niblack's [9] method uses the mean and standard deviation to calculate the threshold. The threshold $T$ of any pixel is given by

$$
T(i, j)=m(i, j)+k \cdot s(i, j)
$$

where $m(i, j)$ and $s(i, j)$ refer to the mean and the variance of the gray values in the neighborhood, respectively. Sauvola's method [10] used a dynamic method to calculate the threshold by using the grayscale average and the standard deviation of the current pixel in the neighborhood. Here $T$ is given by

$$
T(i, j)=m(i, j) \cdot\left[1+k \cdot\left(\frac{s(i, j)}{R}-1\right)\right]
$$

where $m(i, j)$ and $s(i, j)$ are the same as those in Niblack's method. $R$ is the dynamic range of standard deviation and $k$ is the correction factor from 0 to 1 . Sauvola's method does not perform well in very light or dark background. The block size is set according to the character property in the image.

Huang [11] employed a local thresholding method in which Otsu's method is applied on non-overlapping blocks. Gato [12] introduced an adaptive thresholding method in which Niblack's method is utilized to estimate foreground and then sequentially the background region is estimated. B. Gangamma [13] demonstrated bilateral filter and mathematical morphology to restore degraded historical image. Quraishi [14] employed a two-way approach using Particle Swarm Optimization (PSO) and bilateral filter.

B.M. Singh [15] proposed an adaptive binarization method for enhancement of severely degraded document images. The proposed method could deal with degradations that included low contrast, variable background, blur etc. but, the method produced noise where there was a large gap between text lines, high contrast above the text or in the entire image and also in areas where amount of text was comparatively less.

Kale [16] employed a hybrid approach in which first a global thresholding approach is applied (using Iterative Global Thresholding (IGT)) and then IGT is re-applied to areas with detected background noise. Tomar [17] illustrated a technique to extract text from historic images by considering the problem as a blind source separation. It aims to calculate the independent components from a linear mixture of source signals by maximizing a contrast function based on higher order cumulants.

$\mathrm{Lu}$ Di [18] proposed a method for binarization of degraded images to tackle degradations like bleeding-through, uneven illumination and contrast variation. The approach concentrated on the difference in image grayscale contrast in various areas.

In the proposed technique, the threshold value to segment foreground from background is calculated adaptively using Gaussian function. Two filtering methods are used in the pre-processing phase and morphological operations are used in the post-processing phase to further enhance the manuscript text readability. The section 2 in the paper explains the proposed method, its further compared with other methods in section 3 .

\section{Methodology}

\subsection{Proposed Method}

The main goal is to separate the foreground (text) layer from the degraded background(noisy) layer but, manuscripts suff er from a range of damages with some parts of the same manuscript heavily aff ected compared to other parts. Hence, the use of a global thresholding method is generally unsuitable and therefore we employed the 
adaptive Gaussian Thresholding technique to separate the text layer from the noisy layer. We also used a novel dual-filtering method to eliminate noise and make further thresholding more effective. Our methodology consists of three steps- Pre-processing, Adaptive Thresholding and Post-processing. The various outputs after applying every step on manuscript image (shown in Figure 1a) is illustrated (Figure 1).

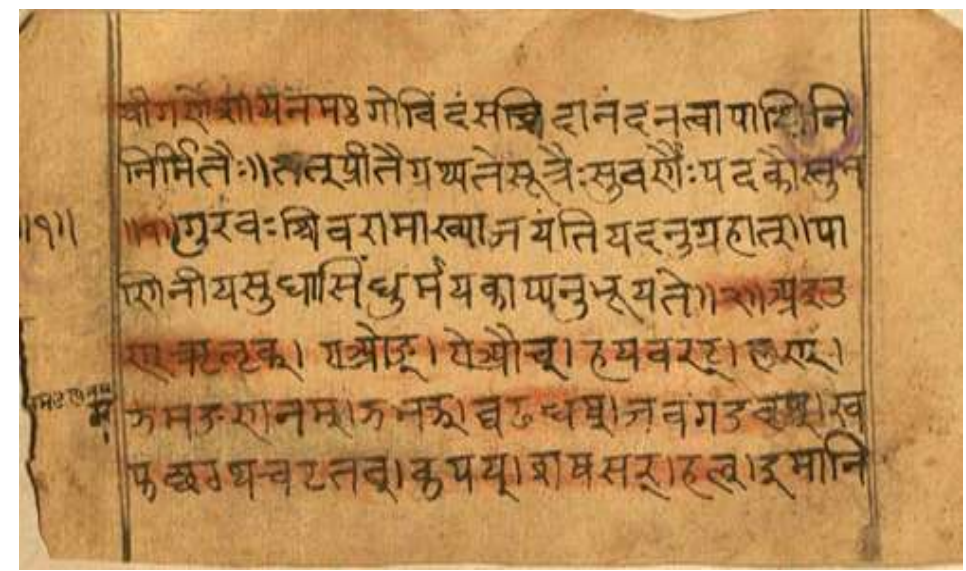

Figure 1a. Original Manuscript

2.1.1. Pre-processing: In order to remove noise and make further processing more efficient, Gaussian filter is used. Gaussian blurring technique convolves each point in an input array with a Gaussian kernel and thereafter sums them all to give the output array. See Figure 1b. A 2D Gaussian can be represented as:

$$
G(x, y)=A e^{-\frac{\left(x-\mu_{x}\right)^{2}}{2 \sigma_{x}^{2}}-\frac{\left(y-\mu_{y}\right)^{2}}{2 \sigma_{y}^{2}}}
$$

where $\mu$ is the mean (the peak) and $\sigma$ represents the variance. The kernel size used was 9 $\times 9$ and $\sigma$ was calculated from kernel size using formula below:

$$
\sigma=0.3 *((\text { kernel size }-1) * 0.5-1)+0.8
$$

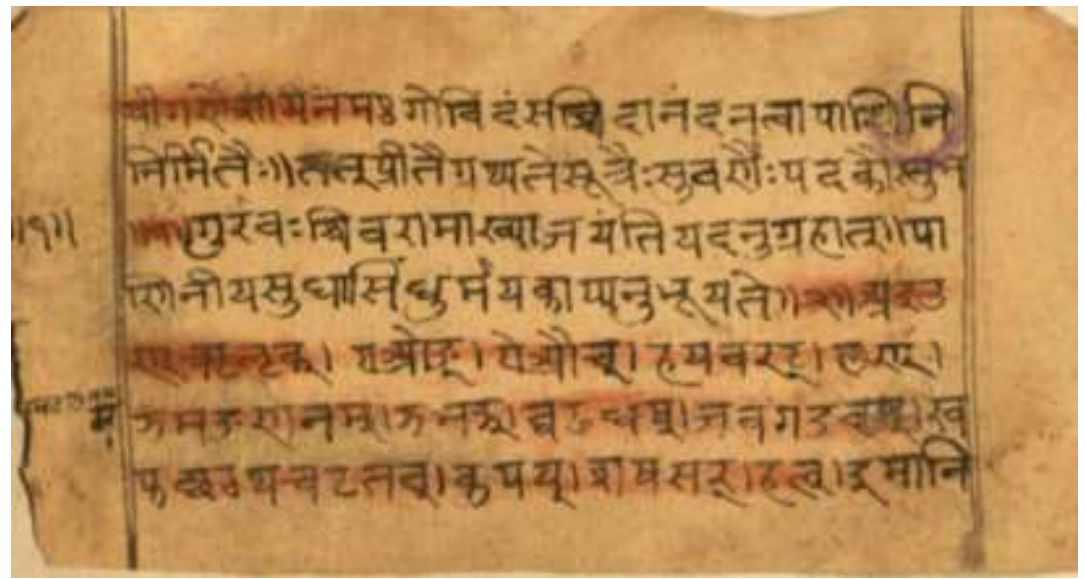

Figure 1b. After Applying Gaussian Blur 
For further smoothing of background surfaces, we employed the mean-shift filter which is the initial step of the meanshift segmentation of an image. D. Comaniciu et. al. [19] expanded the vector $(\mathrm{r}, \mathrm{g}, \mathrm{b})$ that represents each pixel as a 5-dimensional vector $(\mathrm{x}, \mathrm{y}, \mathrm{r}, \mathrm{g}, \mathrm{b})$ which includes location information $(\mathrm{x}, \mathrm{y})$. The mean shift analysis is applied to all points in the image from which we obtain a set of convergence points. After obtaining set of convergence points, we replace the color component of the original point by the color component of the convergence point to generate a new point. The image constructed from the set of new points is the final result of the mean shift analysis. The output is the filtered posterized image with color gradients and fine-grain texture flattened as can be seen in Figure 1c. Mathematically, the meanshift filter works as follows. At every pixel $(\mathrm{X}, \mathrm{Y})$ of the input image the function executes meanshift iterations, i.e., the pixel $(\mathrm{X}, \mathrm{Y})$ neighborhood in the joint space-color hyperspace is considered:

$$
\begin{gathered}
(x, y): X-s p \leq x \leq X+s p \\
Y-s p \leq y \leq Y+s p \\
\|(R, G, B)-(r, g, b)\| \leq s r
\end{gathered}
$$

Where $s p$ is the spatial window radius and $s r$ is the color window radius, $(\mathrm{R}, \mathrm{G}, \mathrm{B})$ and $(\mathrm{r}, \mathrm{g}, \mathrm{b})$ are the vectors of color components at $(\mathrm{X}, \mathrm{Y})$ and $(\mathrm{x}, \mathrm{y})$, respectively (though, the algorithm does not depend on the color space used, so any 3-component color space can be used instead). Over the neighborhood the average spatial value ( $\left.X^{\prime}, Y^{\prime}\right)$ and average color vector (R',G',B') are found and they act as the neighborhood center on the next iteration. After the iterations are over, the color components of the initial pixel (that is, the pixel from where the iterations started) are set to the final value (average color at the last iteration). Also, Gaussian pyramid of multiple levels can be built, and the above procedure is then run on the smallest layer first. After that, the results are propagated to the larger layer and the iterations are run again only on those pixels where the layer colors differ by more than $s r$ from the lower-resolution layer of the pyramid. That makes boundaries of color regions sharper[22]. The value of the parameters we used for the meanshift filter was fairly constant over all images. Both $s p$ and $s r$ were set to 8. Also, Gaussian pyramid of 3 levels was built. The values determined give optimal results for majority of images in our dataset but would need tuning for application to diff erent types of manuscript images.

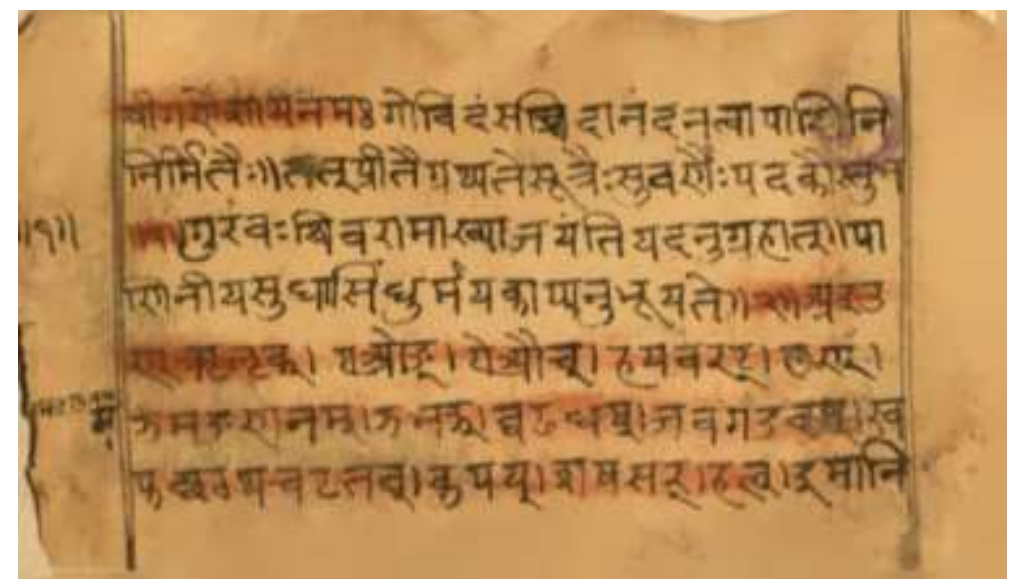

Figure 1c. After Applying Mean Shift Filter

2.1.2. Adaptive thresholding: In this stage, we apply thresholding to segment foreground from background and binarize the image. Image pixels belonging to the foreground areas are detected if the intensity value exceeds a threshold. In our case, we use adaptive 
thresholding to calculate the threshold value such that the value changes in accordance with the neighborhood of the pixel being examined. The threshold value $T(x, y)$ is the weighted sum of the blockSize $\times$ blockSize neighborhood of $(\mathrm{x}, \mathrm{y})$ where weights are calculated using a Gaussian window function $w[n],(x, y)$ is the pixel being considered, blockSize is the size of neighborhood of pixel and $C$ is a constant which is subtracted from the weighted mean calculated. Since the Gaussian function extends to infinity, it must either be truncated at the ends of the window, or itself windowed with another zero-ended window to get the Gaussian window. Since the log of a Gaussian produces a parabola, this can be used for nearly exact quadratic interpolation in frequency estimation [20].

$$
w[n]=e^{-\frac{1}{2}\left(\frac{n-\frac{N-1}{2}}{\frac{\sigma(N-1)}{2}}\right)^{2}}
$$

where $\sigma \leq 0.5$

$\mathrm{N}$ represents the width, in samples, of a discrete-time, symmetrical window function $\mathrm{w}[\mathrm{n}]$, $0 \leq \mathrm{n} \leq \mathrm{N}-1$. The Gaussian window $\mathrm{w}[\mathrm{n}]$ is used to calculate the weights. The blockSize used was 15 and constant $C$ was set to 5 although some amount of variation is required to give optimal results for a particular manuscript image due to variability in the type of manuscript degradation. See Figure 1d.

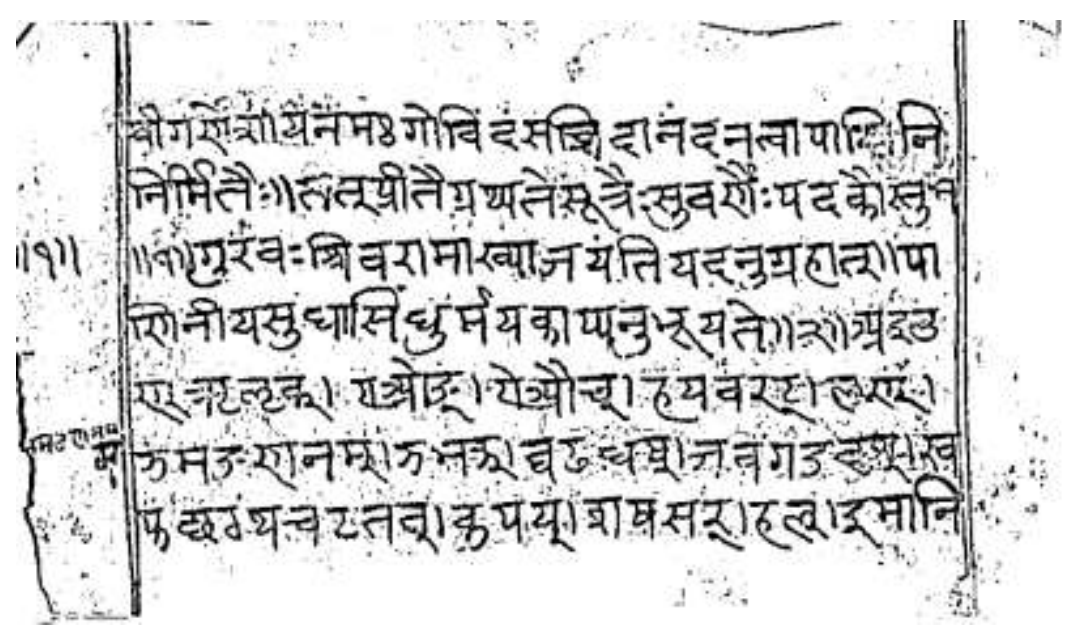

Figure 1d. After Applying Gaussian Thresholding

2.1.3. Post-processing: In this phase morphological operations- dilation and erosion are used, with a $3 \times 3$ rectangular structuring element, to reduce object noise from the binary image and further enhance the readability of the image and give the final result which can be seen in Figure 1e. 


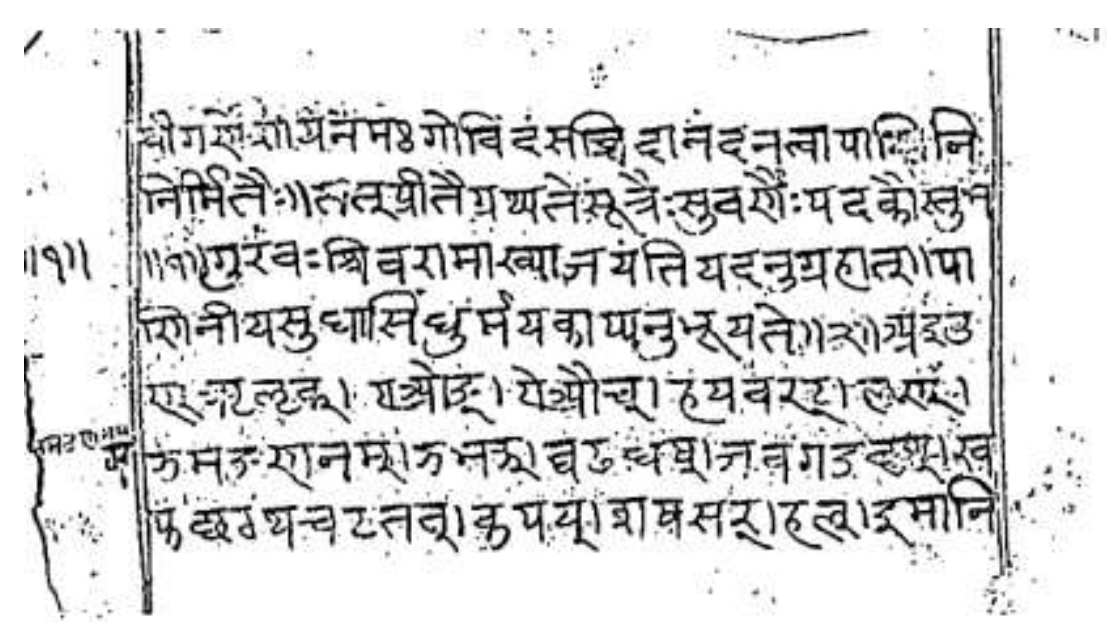

Figure 1e. Final Result After Pre-procesing

\section{Performance Analysis with other Methods}

We have compared the results of our method with that of Bernsen's[8], Niblack's[9], Sauvola's[10] and Gato's[12] methods. The results of the proposed methods were better qualitatively as well as quantitatively. Bernsen's method computes an adaptive threshold by using the maximum and minimum gray-level value of the local block which faces discrete threshold value problems. Niblack's method calculates a local threshold by using the mean and standard deviation value of gray-level image in a local block. In uneven illumination images, Niblack's method usually generates poor quality result. Sauvola's method is essentially an improved Niblack's method such that a variable $r$ is added to Niblack's formula to change behavior from static to dynamic range standard deviation giving better results. Gato's method applies adaptive thresholding to deal with uneven illumination and degraded document images but, does not give satisfactory results when applied to manuscript images. Even though it eliminates noisy background eff ectively it suff ers from non-uniform character formation which makes text legibility an issue when this method is applied, as can be seen in Figure 2d. Our proposed method gains its advantage over other methods from the use of adaptive Gaussian thresholding along with the use of a dual filtering technique which enhances legibility and eff ective binarization of text from background layer. See Figure 1e. The qualitative results that can be seen in Figure 2 correspond to various methods applied on manuscript image shown in Figure 1a. For quantitative estimation we used the well-known measures pseudo F-measure [21], PSNR[23], NRM[23-24], DRD[26], MPM[25] and geometric accuracy[27]. 


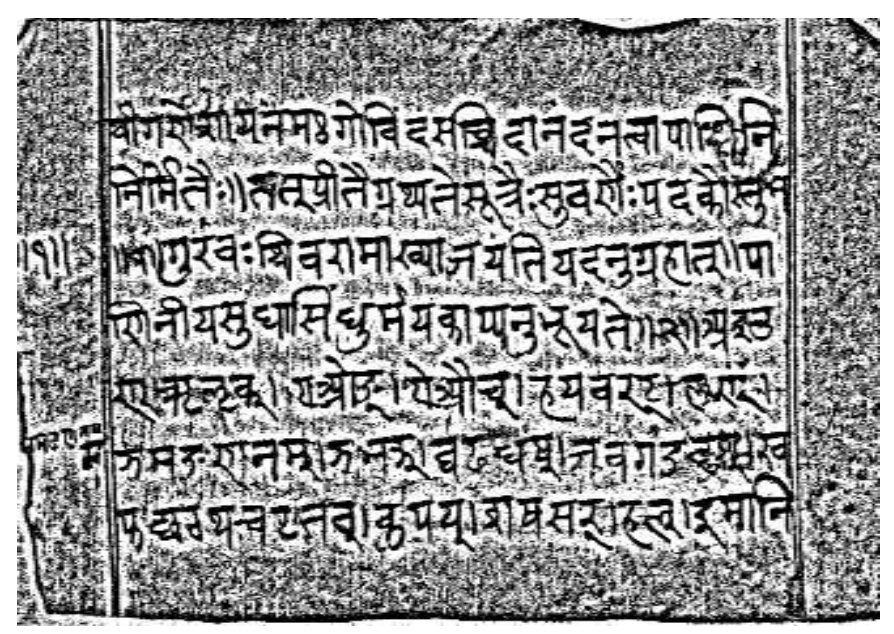

Figure 2a. Result from Bernsen's Method

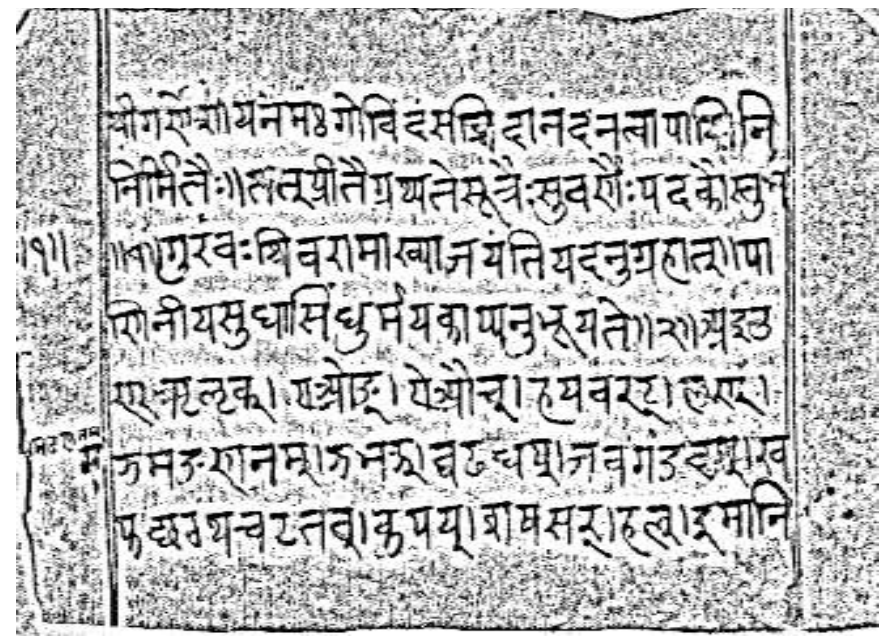

Figure 2b. Result from Niblack's Method

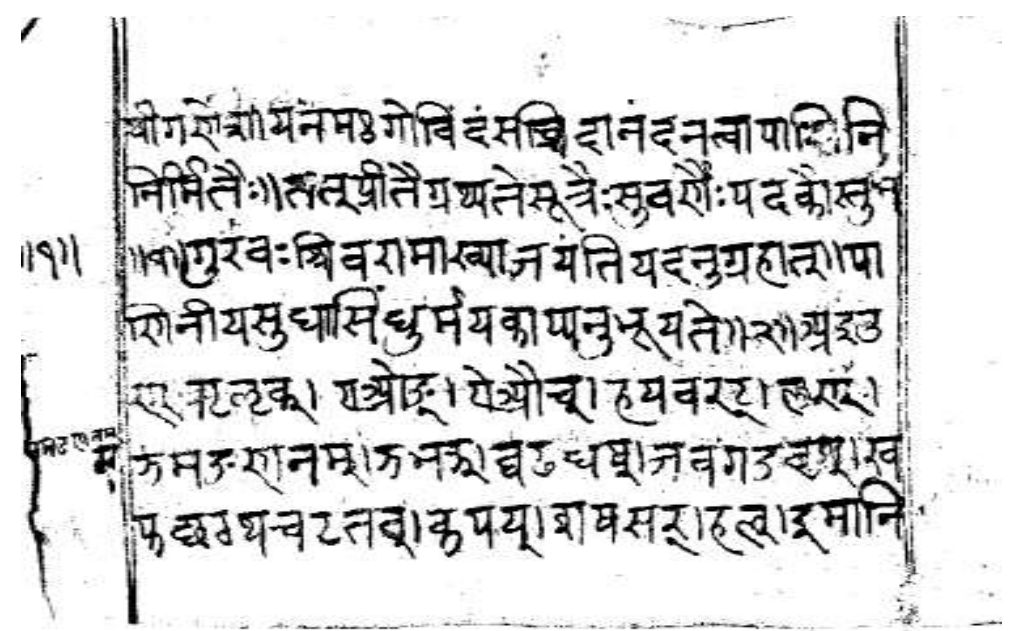

Figure 2c. Result from Sauvola's Method 


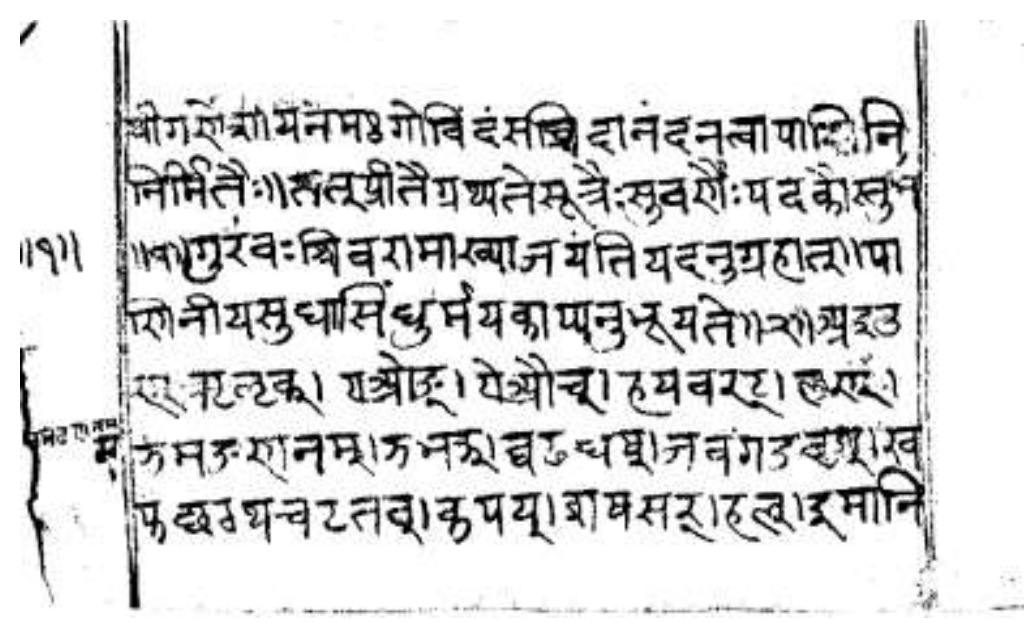

Figure 2d. Result from Gato's Method

\section{Pseudo F-Measure}

$$
=\frac{2 \times \mathrm{p}-\text { Recall } \times \mathrm{p}-\text { Precision }}{\mathrm{p}-\text { Recall }+\mathrm{p}-\text { Precision }}
$$

where p-Recall is the pseudo-Recall and p-Precision is the pseudo-Precision.

PSNR (Peak signal-to-noise ration)

$$
P S N R=10 \log \frac{C^{2}}{M S E}
$$

where $M S E=\frac{\sum_{x=1}^{M} \sum_{y=1}^{N}\left(I(x, y)-I^{\prime}(x, y)\right)^{2}}{M N}$

$\mathrm{C}$ is the difference between the foreground and background. The higher the value of PSNR, the higher the similarity of the two MxN images is.

\section{DRD (Distance reciprocal distortion)}

The distortion of a processed image $\mathrm{g}(\mathrm{x}, \mathrm{y})$ compared with the original image $f(x, y)$ is measured by using a weighted matrix with each of its weights determined by the reciprocal of a distance measured from the center pixel.

$$
D R D=\frac{\sum_{k=1}^{S} \mathrm{DRD}_{k}}{N U B N}
$$

where NUBN is to estimate the valid (non-empty) area in the image and it is defined as the number of non-uniform $8 \times 8$ blocks, $\mathrm{DRD}_{\mathrm{k}}$ is defined as:

$$
D R D_{k}=\sum_{i, j}\left(D_{k}(i, j) * W_{N_{m}}(i, j)\right)
$$

where $\mathrm{W}_{\mathrm{Nm}}$ is the normalized weight matrix and $\mathrm{D}_{\mathrm{k}}$ is given by

$$
D_{k}(i, j)=\left|B_{k}(i, j)-g(x, y)_{k}\right|
$$

Thus, $\mathrm{DRD}_{\mathrm{k}}$ equals to the weighted sum of the pixels of the block $\mathrm{B}_{\mathrm{k}}$ of the original image that differ from the flipped pixel $\mathrm{g}(\mathrm{x}, \mathrm{y})_{\mathrm{k}}$ in the processed image. 


\section{NRM (Negative rate metric)}

The negative rate metric NRM is based on the pixel-wise mismatches between the ground truth image and processed image. It combines the false negative rate $\mathrm{NR}_{\mathrm{FN}}$ and the false positive rate $\mathrm{NR}_{\mathrm{FP}}$. It is denoted as follows:

$$
\begin{array}{r}
N R M=\frac{1}{2}\left(N R_{F N}+N R_{F P}\right) \\
\text { where } \mathrm{NR}_{\mathrm{FN}}=\mathrm{N}_{\mathrm{FN}} /\left(\mathrm{N}_{\mathrm{FN}}+\mathrm{N}_{\mathrm{TP}}\right), \mathrm{NR}_{\mathrm{FP}}=\mathrm{N}_{\mathrm{FP}} /\left(\mathrm{N}_{\mathrm{FP}}+\mathrm{N}_{\mathrm{TN}}\right)
\end{array}
$$

$\mathrm{N}_{\mathrm{TP}}$ denotes the number of true positives, $\mathrm{N}_{\mathrm{FP}}$ denotes the number of false positives, $\mathrm{N}_{\mathrm{TN}}$ denotes the number of true negatives, $\mathrm{N}_{\mathrm{FN}}$ denotes the number of false negatives. Binarization quality is better for lower NRM.

\section{MPM (Misclassification Penalty Metric)}

The Misclassification penalty metric MPM evaluates the prediction against the ground truth on an object-by-object basis. Misclassification pixels are penalized by their distance from the ground truth object's border.

$$
M P M=\frac{1}{2}\left(M P_{F N}+M P_{F P}\right)
$$

where $M P_{F N}=\frac{1}{D}\left(\sum_{i=1}^{N_{F N}} d_{F N}^{i}\right), M P_{F P}=\frac{1}{D}\left(\sum_{j=1}^{N_{F P}} d_{F P}^{j}\right), d_{F N}^{i}$ and $d_{F P}^{j}$ denote the distance of the $i^{\text {th }}$ false negative and the $\mathrm{j}^{\text {th }}$ false positive pixel from the contour of the text in the ground truth image. The normalization factor $D$ is the sum over all the pixel-to-contour distances of the ground truth object. A low MPM score denotes that the algorithm is good at identifying an object's boundary.

The evaluation was performed using the above described evaluation measures. The parameters for the other methods were tuned to give best possible result for a particular image. Table 1 shows the evaluation results for each method applied on the manuscript image shown in Figure 1a based on ground truth created semi-automatically.

Table 1. Evaluation Results

\begin{tabular}{|c|c|c|c|c|c|c|}
\hline Method & $\begin{array}{c}\text { Pseudo F- } \\
\text { measure (\%) }\end{array}$ & PSNR & DRD & NRM & $\begin{array}{c}\text { MPM } \\
(\mathbf{x 1 0 0 0})\end{array}$ & $\begin{array}{c}\text { Geometric } \\
\text { Accuracy }\end{array}$ \\
\hline Bernsen & 16.26 & 8.39 & 20.26 & 0.41 & 64.89 & 0.49 \\
\hline Niblack & 20.06 & 10.09 & 11.65 & 0.41 & 33.17 & 0.47 \\
\hline Sauvola & 23.27 & 11.23 & 7.33 & 0.40 & 2.33 & 0.46 \\
\hline Gato & 32.22 & 9.94 & 12.23 & 0.30 & 4.53 & 0.65 \\
\hline $\begin{array}{c}\text { Proposed } \\
\text { Method }\end{array}$ & 47.87 & 9.70 & 14.51 & 0.07 & 5.53 & 0.92 \\
\hline
\end{tabular}

Table 1 shows the qualitative comparison of diff erent methods. Our proposed method was applied on 27 digital manuscript images, the qualitative results of two can be seen in Figure 3 and Figure 4. The result after applying Gato's method is also shown to illustrate the better suitability of our method compared to Gato's method applied on manuscript image. The quantitative comparison with other methods applied on manuscript image shown in Fig 3a and $4 a$ can be seen in Table 2 and 3, respectively. 


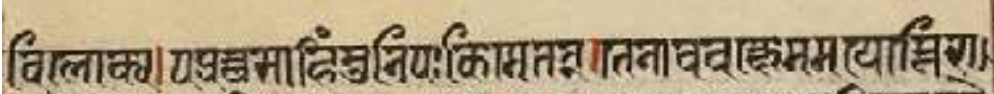

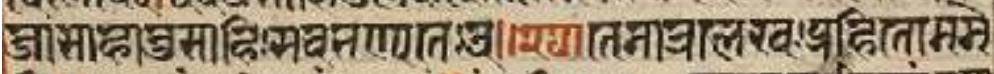

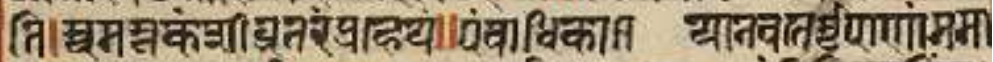

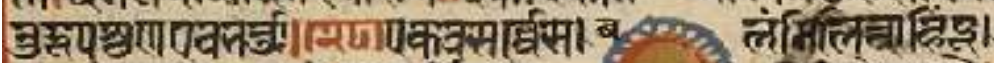

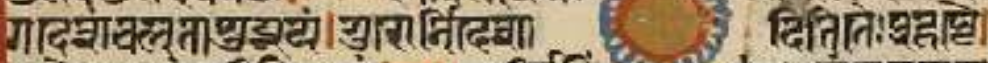

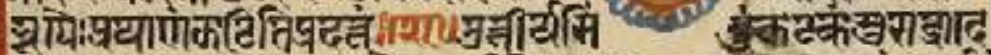

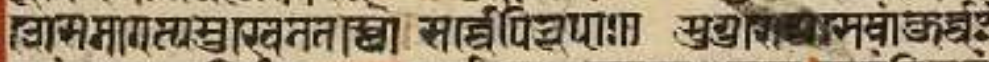

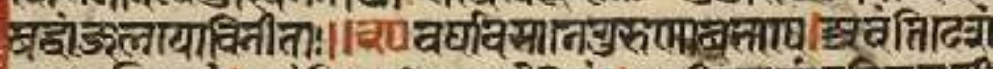

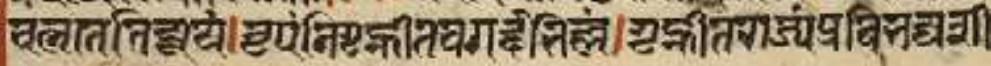

Figure 3a. Original Manuscript Image

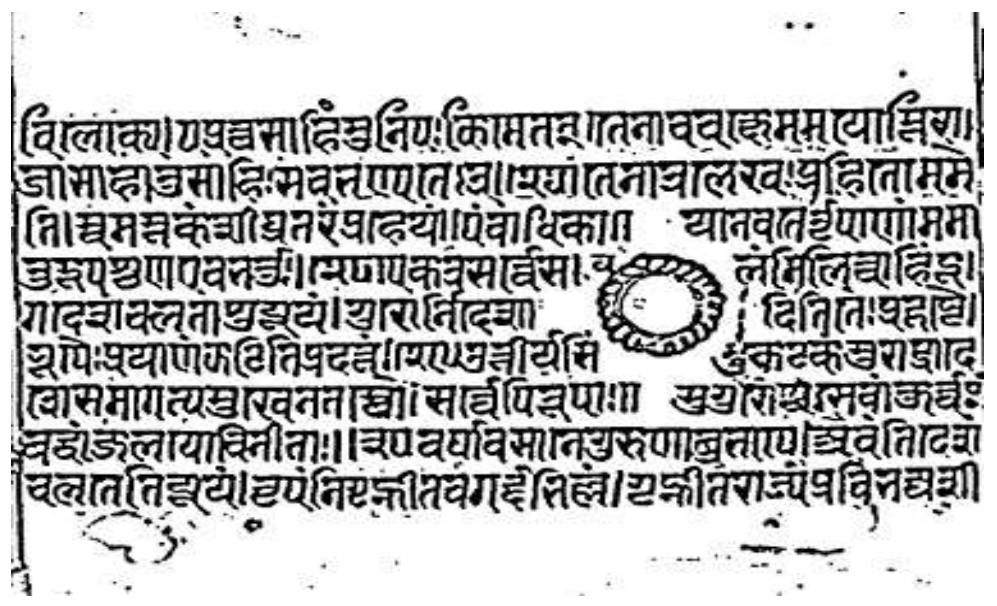

Figure 3b. Result from Proposed Method

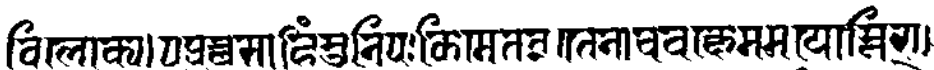

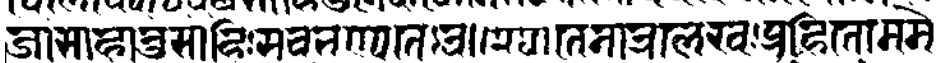

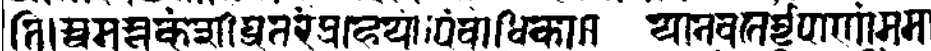

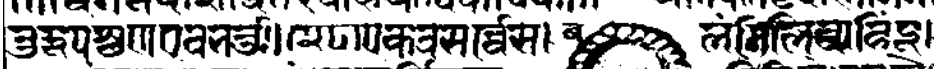

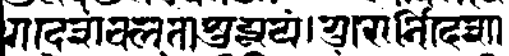

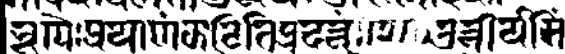

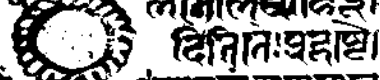

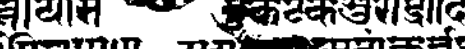

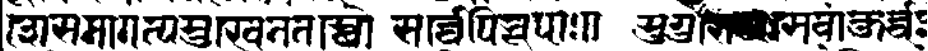

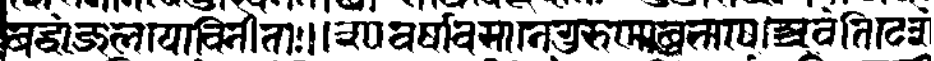

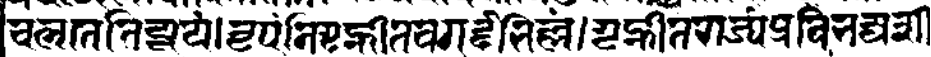

Figure 3c. Result from Gato's Method 
Table 2. Evaluation results

\begin{tabular}{|c|c|c|c|c|c|c|}
\hline Method & $\begin{array}{c}\text { Pseudo F- } \\
\text { measure (\%) }\end{array}$ & PSNR & DRD & NRM & $\begin{array}{c}\text { MPM } \\
(\mathbf{x 1 0 0 0})\end{array}$ & $\begin{array}{c}\text { Geometric } \\
\text { Accuracy }\end{array}$ \\
\hline Bernsen & 38.29 & 7.29 & 13.26 & 0.36 & 63.02 & 0.57 \\
\hline Niblack & 41.03 & 8.04 & 9.87 & 0.35 & 3.21 & 0.56 \\
\hline Sauvola & 47.05 & 8.18 & 9.42 & 0.32 & 2.44 & 0.62 \\
\hline Gato & 61.62 & 9.05 & 7.37 & 0.22 & 2.45 & 0.75 \\
\hline $\begin{array}{c}\text { Proposed } \\
\text { Method }\end{array}$ & 62.17 & 9.31 & 6.58 & 0.23 & 2.60 & 0.74 \\
\hline
\end{tabular}

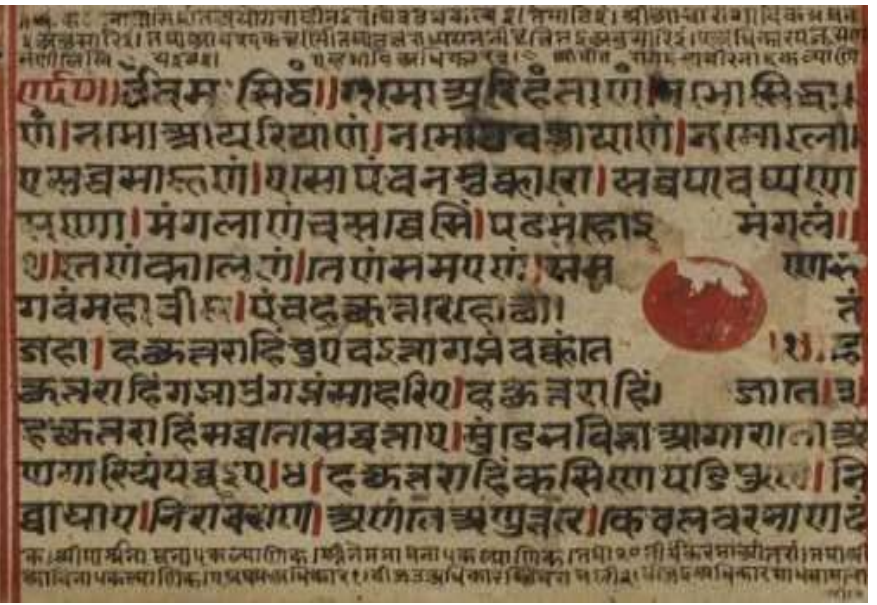

Figure 4a. Original Manuscript Image

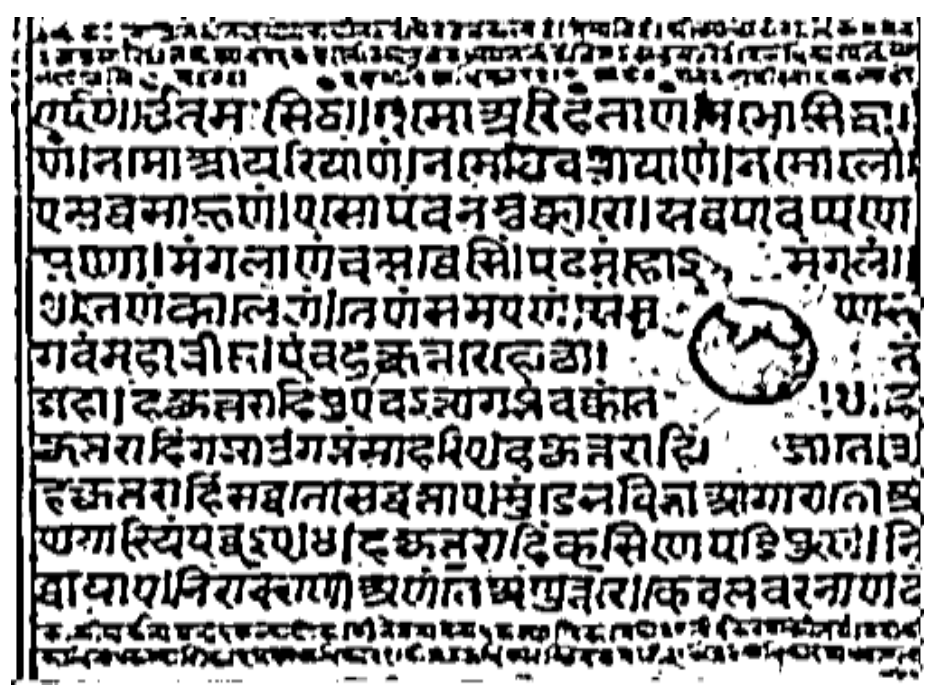

Figure 4b. Result from Proposed Method 


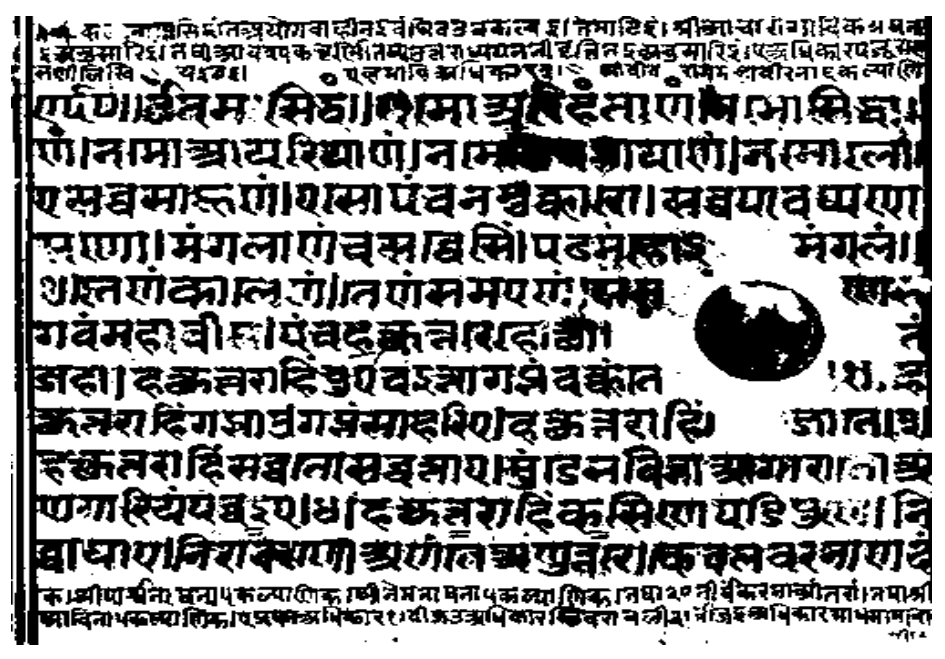

Figure 4c. Result from Gato's Method

Table 3. Evaluation Results

\begin{tabular}{|c|c|c|c|c|c|c|}
\hline Method & $\begin{array}{c}\text { Pseudo F- } \\
\text { measure (\%) }\end{array}$ & PSNR & DRD & NRM & $\begin{array}{c}\text { MPM } \\
(\mathbf{x 1 0 0 0})\end{array}$ & $\begin{array}{c}\text { Geometric } \\
\text { Accuracy }\end{array}$ \\
\hline Bernsen & 28.91 & 6.48 & 7.90 & 0.43 & 18.35 & 0.44 \\
\hline Niblack & 31.85 & 6.82 & 7.00 & 0.41 & 16.41 & 0.46 \\
\hline Sauvola & 28.29 & 6.68 & 7.22 & 0.42 & 11.45 & 0.43 \\
\hline Gato & 53.25 & 6.62 & 8.70 & 0.28 & 30.47 & 0.70 \\
\hline $\begin{array}{c}\text { Proposed } \\
\text { Method }\end{array}$ & 65.02 & 8.11 & 5.89 & 0.21 & 23.17 & 0.78 \\
\hline
\end{tabular}

The quantitative results for all methods applied on our dataset of 27 images can be seen in Table 4. Our method has improved uniform character formation compared to other methods although, in some manuscript images it suffers from inability to eliminate some background noise which manifests itself in the form of small black dots which can be seen in Figures $1 \mathrm{e}$ and $4 \mathrm{~b}$. This inability to eliminate all noisy pixels is a minor drawback in our method but, the improved character formation is a major advantage and could lead to better OCR readability compared to other methods which can be tested as future work.

Table 4. Evaluation Results

\begin{tabular}{|c|c|}
\hline Method & Pseudo F-measure(\%) \\
\hline Bernsen & 27.54 \\
\hline Niblack & 30.21 \\
\hline Sauvola & 32.78 \\
\hline Gato & 50.05 \\
\hline Proposed Method & 60.12 \\
\hline
\end{tabular}

\section{Conclusion}

A novel technique for enhancing and binarizing digital manuscript images has been proposed which is shown to have much improved readability of characters. Our method is much more suitable for enhancing manuscripts compared to other document enhancing methods due to their unsuitability for use in damaged manuscript images. It also performs much better compared to other well-known methods qualitatively as well as 
quantitatively. Our work can be further expanded for enhancing other types of documents with different types of degradation for better OCR readability. However, our method suff ers the drawback of not being able to eliminate noise completely and hence, as future work further modifications to the proposed method could be tested.

\section{References}

[1] J. M. C. Sousa, J. M. Gil, C. S. Ribeiro and J. R. C. Pintom, "Old Document Recognition Using Fuzzy Methods", International Journal of Intelligent Systems Technologies and Applications, vol. 1, no. 3/4, (2006), pp. 263-279.

[2] Y. Leydier, F. Lebourgeois and H. Emptoz, "Text search for medieval manuscript images", Pattern Recognition, vol. 40, no. 12, (2007), pp. 3552-3567.

[3] L. L. Sulem and M. Sigelle, "Recognition of degraded characters using dynamic Bayesian networks", Pattern Recognition, vol. 41, no. 10, (2008), pp. 3092-3103.

[4] S. R. Yahya, S. N. H. S. Abdullah, K. Omar, M. S. Zakaria and C. Y. Liong, "Review on Image Enhancement methods of Old Manuscripts with Damaged background", International Journal on Electrical Engineering and Informatics, vol. 2, no. 1, (2010).

[5] N. Otsu, "A threshold selection method from gray-level histogram," IEEE Transactions on Systems, Man, and Cybernetics, vol. 9, (1979), pp. 62-66.

[6] B. J. N. Kapur, P. K. Sahoo and A. K. C. Wong, "A new method for gray-level picture thresholding using the entropy of the histogram", Computer Vision, Graphics, and Image Processing, vol. 29, (1985), pp. 273-285.

[7] J. Kittler and J. Illingworth, "Minimum error thresholding”, Pattern Recognition, vol. 19, (1986), pp. 4147.

[8] J. Bernsen, "Dynamic thresholding of gray-level images", in Proceeding of the 8th International Conference on Pattern Recognition, (1986), pp. 1251-1255.

[9] W. Niblack, "An Introduction to Digital Image Processing”, Strandberg, Denmark, (1986).

[10] J. Sauvola and M. Pietikainen, "Adaptive document image binarization", Pattern Recognition, vol. 33, (2000), pp. 225-236

[11] Q. Huang, W. Gao and W. Cai, "Thresholding technique with adaptive window selection for uneven lighting image", Pattern Recognition Letter, vol. 26, (2005), pp. 801-808.

[12] B. Gatos, I. Pratikakis and S. Perantonis, "Adaptive degraded document image binarization", Pattern Recognition, vol. 39, (2006), pp. 317-327.

[13] B. Gangamma, K. S. Murthy and A. Vikas Singh, "Restoration of Degraded Historical Document Image", Journal of Emerging Trends in Computing and Information Sciences, vol. 3, no. 5, (2012).

[14] I. Quraishi, M. De, K. G. Dhal, S. Mondal and G. Das "A Novel Hybrid Approach To Restore Historical Degraded Documents", ISSP, vol. 1, (2013), pp. 185-189.

[15] B. M. Singh, R. Sharma, D. Ghosh and A. Mittal, "Adaptive binarization of severely degraded and nonuniformly illuminated documents", International Journal on Document Analysis and Recognition (IJDAR), vol. 17, no. 4, (2014), pp. 393-412

[16] Kale, Gandhe, Phade and Dhulekar, "Enhancement of Old Images and documents by Digital Image Processing techniques", ICCICT, (2015).

[17] A. Tomar, A. Raj, S. Chaudhury and S. Indu, "Enhancement and Retrieval of Historic Inscription Images", In ComputerVision-ACCV 2014 Workshops, Springer International Publishing, (2014).

[18] D. Lu, X. Huang, C. Liu, X. Lin, H. Zhang and J. Yan, "Binarization of degraded document image based on contrast enhancement", Proceedings of the $35^{\text {th }}$ Chinese Control Conference, Chengdu, China, (2016).

[19] D. Comaniciu and P. Meer, "Mean Shift: A robust approach toward feature space analysis", IEEE Transactions on Pattern Analysis and Machine Intelligence, (2002).

[20] Gaussian Window and Transform, ccrma.stanford.edu, (2016).

[21] K. Ntirogiannis, B. Gatos and I. Pratikakis, "Performance Evaluation Methodology for Historical Document Image Binarization”, IEEE Transactions On Image Processing, vol. 22, no. 2, (2013).

[22] http://docs.opencv.org/3.0beta/modules/imgproc/doc/filtering.html\#pyrmeanshiftfiltering

[23] I. Pratikakis, B. Gatos and K. Ntirogiannis, "H-DIBCO 2010 - handwritten document image binarization competition", in Proceedings Int. Conf. Frontiers Handwrit. Recognit., Kolkata, India, (2010), pp. 727732.

[24] B. Gatos, K. Ntirogiannis and I. Pratikakis, "DIBCO 2009: Document image binarization contest", International Journal Document Anal. Recognit., vol. 14, no. 1, (2011), pp. 35-44.

[25] D. P. Young and J. M. Ferryman, "PETS Metrics: On-Line Performance Evaluation Service", ICCCN '05 Proceedings of the 14th International Conference on Computer Communications and Networks, (2005), pp. 317-324.

[26] H. Lu, A. C. Kot and Y. Q. Shi, "Distance-reciprocal distortion measure for binary document images", IEEE Signal Process. Lett., vol. 11, no. 2, (2004), pp. 228-231. 
[27] R. Paredes, E. Kavallieratou and R. D. Lins, "ICFHR 2010 contest: Quantitative evaluation of binarization algorithms", in Proceedings Int. Conf. Frontiers Handwrit. Recognit., Kolkata, India, (2010), pp. $733-736$.

\section{Authors}

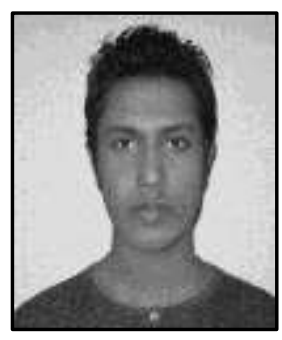

Nikunj Khetan, he is a final year undergraduate student from Delhi Technological University majoring in Computer Science. His research interests include Computer Vision, Image Processing and Machine Learning.

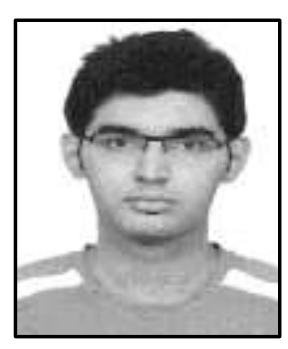

Lakshya Kejriwal, he is a final year undergraduate student from Delhi Technological University majoring in Software Engineering. His research interests include Computer Vision, Image Processing and Deep Learning.

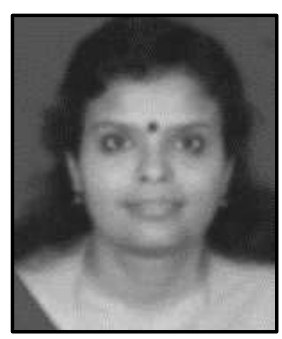

Sreedevi Indu, she received her B.Tech and M.Tech degrees from the University of Kerala and her Ph.D. from Delhi University. She is currently the Head of Electronics \& Communication Engineering Department of Delhi Technological University. Her research interests include Computer Vision, Image Processing and Wireless Sensor Networks. 